\title{
Infections inapparentes chez l'Homme : un cheval de Troie pour l'introduction et la diffusion des arbovirus transmis par des moustiques dans les régions non endémiques?
}

\author{
Asymptomatic infections in man: a Trojan horse for the introduction \\ and spread of mosquito-borne arboviruses in non-endemic areas?
}

\author{
C. Chastel \\ Reçu le 14 décembre 2010 ; accepté le 5 avril 2011 \\ (C) Société de pathologie exotique et Springer-Verlag France 2011
}

Résumé Dans les infections à arbovirus transmises par des moustiques, les formes inapparentes chez l'Homme sont beaucoup plus fréquentes que les formes apparentes, mais leur rôle réel dans l'introduction et la diffusion ultérieure de ces infections dans les régions non endémiques reste à préciser. Nous avons recueilli les données utiles à la compréhension de ce problème dans la littérature scientifique, de 1952 à 2010, grâce à Pubmed et à d'autres sources bibliographiques. Ces données ont été analysées dans l'optique de déterminer si, dans les infections inapparentes humaines à arbovirus, la virémie pouvait être suffisante pour constituer un risque réel d'introduction de ces infections dans des régions jusque-là indemnes. Au cours des épidémies de dengue et d'infections à virus chikungunya (CHIKV), on pense que l'Homme est le seul hôte vertébré concerné. Puisqu'un très grand nombre d'individus sont infectés et puisque les taux de virémie varient considérablement chez les malades symptomatiques, il est raisonnable de penser que, dans un certain nombre de formes inapparentes, le taux de virémie pourrait être suffisant pour infecter des moustiques compétents. Par ailleurs, dans la dengue et les infections à CHIKV, des infections nosocomiales ont été identifiées, représentant un mode alternatif d'introduction des virus dans des régions indemnes. Dans les arboviroses zoonotiques transmises par des moustiques comme l'encéphalite japonaise ou les infections à virus West Nile (WNV), la situation est bien différente puisque l'Homme est considéré comme une impasse épidémiologique. Toutefois, si l'on tient compte du très grand nombre de formes inapparentes survenant chez l'Homme au cours des épidémies et de l'existence de modes de contamination nouveaux récemment identifiés (transfusion sanguine, greffe d'organes, voie

C. Chastel $(\bowtie)$

3, rue Rouget-de-1'Isle, F-29200, Brest, France

e-mail : chastelc@aol.com transplacentaire), l'introduction de ces arboviroses peut ainsi être assurée dans des régions jusque-là épargnées. Pour citer cette revue : Bull. Soc. Pathol. Exot. 104 (2011).

Mots clés Arbovirus transmis par des moustiques . Infections inapparentes chez l'Homme · Risques d'introduction en région non endémique $\cdot$ Dengue . Chikungunya $\cdot$ Encéphalite japonaise $\cdot$ West Nile $\cdot$ Fièvre de la Vallée du Rift

Abstract In mosquito-borne arbovirus infections in man the asymptomatic cases are much more frequent than the symptomatic ones, but their true role in the introduction and subsequent spread of such diseases in non-endemic areas remains to be clarified. We have collected pertinent data from English and French literature from 1952 to 2010 through Pubmed and other bibliographic sources. Data were analysed to assess if viremia in asymptomatic human arbovirus infections might be sufficient to represent a true risk for introduction in non-endemic areas. During dengue and chikungunya fever outbreaks, humans are believed to be the only vertebrate hosts. Since a very large number of individuals are infected and since viremic levels are known to vary by many orders of magnitude in symptomatic patients, it is reasonable to augur that a proportion of asymptomatic cases might reach levels of viremia sufficient to infect competent mosquitoes. Moreover, in both dengue and chikungunya fever, nosocomial infections have been identified representing an alternative opportunity for virus introduction in non-endemic areas. In zoonotic mosquito-borne arbovirus infections such as Japanese encephalitis or West Nile infection, the situation is quite different since humans are considered as "dead-end" hosts. However, the very large number of asymptomatic cases arising during outbreaks and the existence of newly recognised ways of contamination (blood transfusion, organ transplantation, transplacental way etc.) 
may also ensure their introduction and subsequent spread in new areas. To cite this journal: Bull. Soc. Pathol. Exot. 104 (2011).

Keyword Mosquito-borne arboviruses · Asymptomatic infection in man - Risks for introduction in non-endemic areas $\cdot$ Dengue $\cdot$ Chikungunya $\cdot$ Japanese encephalitis . West Nile $\cdot$ Rift Valley fever

\section{Introduction}

$\mathrm{Au}$ cours des dernières décennies, plusieurs arbovirus transmis par des moustiques ont envahi des régions géographiques où ils n'avaient jamais été observés. Ils y ont provoqué des épidémies plus ou moins extensives et sévères. Plusieurs facteurs épidémiologiques ont pu favoriser ces invasions, en particulier l'accroissement du trafic aérien international, l'augmentation continue de la population mondiale et l'urbanisation galopante $[15,16]$, tandis que les bouleversements climatiques constituaient des facteurs aggravants [13,24,51]. L'ampleur sans précédent de ce phénomène fait que l'on peut se poser la question de savoir quelle a pu être la part des infections inapparentes (asymptomatiques) dans l'introduction et la diffusion récente des arbovirus transmis par des moustiques, dans des régions jusque-là épargnées. Cette revue a pour but d'examiner la vraisemblance de l'hypothèse selon laquelle les formes inapparentes ont pu y jouer un rôle réel.

\section{Exemples récents d'infections à arbovirus transmis par des moustiques ayant envahi des régions jusque-là indemnes}

En 1999, le virus West Nile (WNV), jusque-là cantonné à l'Ancien Monde, a été introduit aux États-Unis à partir du Moyen-Orient [35]. Il a ensuite colonisé rapidement une grande partie du Nouveau Monde, y provoquant des dizaines de milliers d'infections humaines et animales, avec de nombreux cas d'encéphalite chez des gens âgés [15].

Jusqu'en 2000, le virus de la Vallée du Rift (RVFV) était uniquement connu du continent africain. Mais, à cette date, il a franchi la mer Rouge, diffusant de manière brutale, épizootique et épidémique, en Arabie saoudite et au Yémen [38]. Plus récemment, il a gagné l'île de Mayotte, dans l'océan Indien [58].

À l'automne 2001, le virus Usutu (USUV), un Flavivirus originaire d'Afrique tropicale et proche de WNV, a brusquement émergé en Autriche. Il y a provoqué d'importantes mortalités chez des oiseaux sauvages ou hébergés en zoo dans la région de Vienne $[68,69]$. Cette situation rappelait celle déjà observée aux États-Unis après l'introduction de WNV en 1999. Ce virus s'est ensuite étendu à plusieurs pays d'Europe, de la Hongrie à l'Espagne et du RoyaumeUni à l'Italie du nord-est. Dans cette dernière région, il y a circulé en même temps que le WNV, entraînant quelques infections humaines chez des transfusés ou des immunodéprimés [9].

En 2005, le virus de l'encéphalite japonaise (JEV), ayant progressé d'île en île à travers le détroit de Torres [28,53], a réussi à s'infiltrer dans le Nord-est de l'Australie, trouvant sur place des moustiques compétents $[65,66]$.

Encore plus spectaculaire fut l'épidémie sans précédent qui s'est abattue, à partir de 2005, sur plusieurs îles du sud de l'océan Indien, provoquée par le virus chikungunya (CHIKV). Rien qu'à La Réunion, plus du tiers de la population fut atteint, présentant parfois des formes cliniques graves jusque-là inconnues, parfois mortelles. Le vecteur était Aedes albopictus dont on ne soupçonnait pas qu'il puisse posséder une telle compétence vectorielle pour ce virus $[14,23,48,55]$. De l'océan Indien, le virus a gagné l'Inde méridionale, où il a entraîné au moins 1,5 million d'infections humaines [11,34,43,50], avant d'être propulsé vers l'Italie du nord-est, y provoquant la première épidémie autochtone de chikungunya (205 cas) en Europe [52].

Beaucoup plus anecdotique, encore que peut-être chargée de lourdes menaces pour l'avenir, est l'arrivée inopinée en 2007 du virus Zika (ZIKV) sur l'Île de Yap en Fédération de Micronésie. Provenant vraisemblablement du Sud-est asiatique, véhiculé sur place par Aedes hensilli, ce virus a rapidement infecté les trois quarts de la population [20,37]. C'était la première incursion de ce Flavivirus dans la région.

Enfin, ce que l'on redoutait depuis quelque temps en France s'est produit en septembre 2010 : deux cas de dengue et deux cas d'infections à CHIKV, tous autochtones, ont été diagnostiqués dans le Var et dans les Alpes-Maritimes. Comme à La Réunion ou en Émilie-Romagne, le vecteur responsable est $A$. albopictus, maintenant bien implanté dans la région $[6,40]$.

\section{Mesures prises pour tenter de limiter ces introductions}

Il est impossible d'empêcher un voyageur très légèrement fébrile et, a fortiori, totalement asymptomatique, mais infecté, de débarquer dans un aéroport international. Or, il peut provenir d'une région où sévit une épidémie et être virémique au débarquement, constituant une source potentielle d'autres infections.

Au cours des infections à arbovirus transmises par des moustiques, les formes cliniques discrètes, voire totalement inapparentes sont très fréquentes. C'est le cas pour les infections provoquées par les virus de la dengue (DENV), de l'encéphalite japonaise (JEV), de chikungunya (CHIKV), de la fièvre de la Vallée du Rift (RVFV) et de West Nile 
(WN), tous susceptibles d'être introduits dans d'autres régions du monde à partir de leurs foyers naturels. Dans le but de détecter rapidement d'éventuels cas de dengue introduits à Taïwan, les autorités sanitaires ont établi un programme intégré de surveillance, associant l'information des passagers, la détection des passagers fiévreux par infrarouge et le recours à un réseau de laboratoires de virologie capables d'effectuer des tests rapides. Cette stratégie, appliquée entre juillet 2003 et juin 2004, a permis d'identifier 40 cas de dengue par RT-PCR, tous virémiques [56]. Cette surveillance, forcément coûteuse, nécessite de disposer d'infrastructures et de personnel performants, ce qui est loin d'être partout le cas. De plus, la thermométrie cutanée IR ne peut pas repérer les sujets infectés, non fébriles.

\section{Formes inapparentes des arboviroses transmises par des moustiques : importance numérique}

On séparera celles dont l'hôte principal est l'Homme (anthropozoonoses) de celles qui sont strictement des zoonoses, bien que dans les deux cas les formes inapparentes soient nombreuses (Tableau 1).

\section{Anthropozoonoses arbovirales transmises par des moustiques : chikungunya et dengue}

Pendant les épidémies, l'Homme y est l'hôte vertébré principal, sinon exclusif. Au cours des épidémies provoquées par CHIKV, entre 2004 et 2006, plusieurs travaux ont établi la

\begin{tabular}{|c|c|c|c|}
\hline Virus & Genre & Ratio $\mathrm{I} / \mathrm{A}^{\mathrm{a}}$ & Références \\
\hline CHIKV & Alphavirus & $0,03: 1$ à $3: 1$ & {$[34,49,57]$} \\
\hline DENV & Flavivirus & $1: 1$ à $13: 1$ & {$[4,8,21,70]$} \\
\hline WNV & Flavivirus & $2,9: 1$ à $4,4: 1$ & {$[42,67,71]$} \\
\hline JEV & Flavivirus & $25: 1$ à $1000: 1$ & {$[25,60,61]$} \\
\hline YFV & Flavivirus & 1,5:1 à 9:1 & {$[18]$} \\
\hline SLEV & Flavivirus & $61: 1$ & {$[7]$} \\
\hline MVEV & Flavivirus & 500 à 1000:1 & {$[2]$} \\
\hline RVFV & Phlebovirus & $8,2: 1$ & {$[33]$} \\
\hline
\end{tabular}

proportion des formes inapparentes. À La Réunion, le pourcentage de ces dernières a été estimé à $3,2 \%$, soit un ratio infections inapparentes (I)/infections apparentes (A) de 0,03:1 [49]. Mais en Inde du sud, ce ratio fut trouvé plus élevé, soit 1:1 [34], ainsi qu'à Mayotte, 3:1 [57]. Ainsi, le nombre réel de cas survenus lors de ces deux dernières épidémies était, en réalité, le double ou le quadruple de ce que pouvait indiquer le dénombrement des seuls cas cliniquement avérés.

Les quatre DENV, dont on pense qu'ils provoquent, chaque année, environ 100 millions d'infections apparentes [27], nous offrent d'autres exemples de l'importance numérique des infections inapparentes qu'ils induisent. Au cours d'une enquête prospective réalisée en 1980-1981, à Bangkok, chez des enfants scolarisés, les auteurs ont établi un ratio I/A de 6,1:1. Mais lorsqu'ils ont calculé ce même ratio en fonction du type de virus infectant, ce ratio était de 5,5:1 pour le DENV 1 et de 4,5:1 pour le DENV 2. Quant aux infections à DENV 4, elles étaient toutes asymptomatiques [8]. Mais, dans une autre enquête prospective conduite dans le nord de la Thaïlande, à Kamphaeng Phet, entre 1998 et 2000, les résultats ont été sensiblement différents, le ratio n'étant plus que 1,1:1 [21]. Par ailleurs, une enquête similaire a été réalisée au Nicaragua, entre 2001 et 2003, chez des enfants scolarisés de 4 à 16 ans et vivant dans la ville de Managua. Elle a montré qu'en 2001, année où le DENV 2 était dominant, ce ratio atteignait 13:1, tandis qu'il tombait à 6,1:1 en 2002, année où DENV 1 était le sérotype le plus souvent isolé [4]. Finalement, à Singapour, les autorités sanitaires ont retenu un ratio se situant entre $2: 1$ et $10: 1$, soit un risque majeur pour les receveurs d'une transfusion sanguine [70].

Ces différences régionales peuvent s'expliquer par des variations individuelles de réceptivité aux DENV ou par une virulence différente des souches virales en circulation [4]. D'ailleurs, l'épidémiologie de la dengue pourrait être différente dans le Sud-est asiatique et dans les Amériques [26].

\section{Zoonoses arbovirales transmises par des moustiques}

L'apparition soudaine du WNV à New York, en 1999, et sa diffusion rapide dans les Amériques ont conduit à une meilleure connaissance de l'épidémiologie et des capacités adaptatives de ce Flavivirus à de nouveaux biotopes, à une faune sauvage nouvelle et variée et aux espèces locales de moustiques. De plus, les infections humaines que ce virus a entraînées dans le Nouveau Monde étaient surtout des atteintes du système nerveux central chez des gens âgés, et des modes de transmission jusque-là ignorés ont été reconnus : transfusion sanguine, transplantation d'organes, hémodialyse, voie transplacentaire [15]. Enfin, il a été démontré que les infections inapparentes à WNV étaient particulièrement fréquentes. 
Ainsi, lors des épidémies qui ont frappé la région métropolitaine de New York et l'État du New Jersey, un ratio I/A de 3,8:1 à 4,4:1 a été établi [42,67,71]. Chez des donneurs de sang, la virémie a pu être décelée jusqu'au $15^{\mathrm{e}}$ jour. Dans une autre enquête réalisée par la Croix-Rouge américaine, chez 1436 donneurs de sang, ce ratio était de 2,9:1 [71].

Le JEV est un Flavivirus transmis par des moustiques et appartenant au même complexe génomique que le WNV. Il est très largement répandu en Asie tropicale, en ExtrêmeOrient et en Indonésie [22]. Entre 1995 et 1998, il a été repéré dans les îles du détroit de Torres, séparant le Nordest de l'Australie de la Nouvelle-Guinée $(170 \mathrm{~km})$. Puis, en 1998, on l'a identifié au Cap York. Il y serait parvenu par des moustiques du genre Culex, poussés par les vents [22].

On sait de longue date que les infections inapparentes par JEV sont beaucoup plus fréquentes que les infections apparentes, tant chez l'Homme que chez le porc domestique. Dans les années 1950, les enfants japonais vivant dans des zones d'endémie étaient fréquemment infectés et un ratio I/A de 500:1 à 1000:1 avait alors été retenu [60,61]. Par contre, pendant l'épidémie qui a frappé la Corée du Sud, en 1958, un ratio de seulement 25:1 a été établi pour le personnel résidant de l'armée américaine [25]. Par ailleurs, plusieurs cas d'encéphalite japonaise ont été diagnostiqués en Scandinavie et aux États-Unis, chez des voyageurs contaminés en Indonésie ou dans le Sud-est asiatique [10,46].

Le virus de la fièvre jaune (YFV) continue à représenter une grave menace pour l'humanité. Alors qu'il existe un excellent vaccin contre cette arbovirose (17D-204), des cas sporadiques de contact avec la forêt pluviale surviennent continuellement en Amérique latine, alors que des cas sporadiques et des épidémies sont observés en Afrique tropicale, provoquant chaque année des centaines de morts [17]. De plus, des voyageurs non vaccinés provenant d'Europe ou des États-Unis s'infectent dans ces foyers naturels avec un risque élevé d'en mourir.

$\mathrm{Au}$ cours des épidémies de fièvre jaune, il y a de 60 à $90 \%$ d'infections inapparentes, ce qui correspond à des ratios $\mathrm{I} / \mathrm{A}$ de 1,5:1 à 9:1 [18]. Pour les autres infections zoonotiques à Flavivirus, des ratios de 61:1 pour le virus de l'encéphalite de Saint-Louis (SLEV) en Amérique du Nord [7] et de 500:1 à 1000:1 pour le virus de l'encéphalite de la Murray Valley (MVEV) en Australie [2] ont été publiés.

Le RVFV, un Phlebovirus, est connu depuis 1912 comme responsable de plusieurs épizooties et épidémies dévastatrices en Afrique, à Madagascar $[3,39,41,54]$ et en Péninsule arabique [38]. Au cours de la première épidémie ayant atteint la Mauritanie, en 1987, un ratio I/A de 8,2:1 a été calculé chez l'Homme [33]. Mais, lorsque le RVFV est réapparu en Égypte, en 1993, on a dénombré 23 infections, toutes inapparentes, chez 1181 travailleurs des abattoirs, un personnel à haut risque d'être contaminé par des animaux malades [1].

\section{Risques épidémiologiques représentés par des infections inapparentes}

On sait maintenant que la transfusion sanguine, les greffes d'organes, l'hémodialyse, les piqûres accidentelles par des aiguilles souillées, peut-être l'allaitement maternel, peuvent représenter des risques de propagation d'arbovirus transmis par des moustiques. C'est le cas pour les DENV [45], le WNV [31], le CHIKV [5] et l'USUV [9]. C'est pourquoi, lorsque l'épidémie de chikungunya s'est brusquement étendue à La Réunion (2006), on a décidé d'arrêter l'activité des banques locales de sang. Le sang et les produits sanguins furent alors transitoirement importés de la métropole. Il a également été prouvé que des cornées recueillies chez des donneurs asymptomatiques pouvaient être contaminées par le CHIKV [5].

La voie transplacentaire peut aussi être empruntée par certains de ces virus (DENV, WNV, CHIKV), même si la contamination de l'enfant est plus fréquemment périnatale que placentaire $[23,63]$.

Dans les régions non endémiques, des arbovirus transmis par des moustiques sont introduits continuellement par des voyageurs asymptomatiques, mais virémiques. Pour que de telles introductions puissent conduire ensuite à des infections autochtones et, éventuellement, à une épidémie, certaines conditions doivent impérativement être remplies :

- les voyageurs infectés doivent présenter une virémie suffisamment élevée pour être capable d'infecter les espèces locales de moustiques ;

- ces moustiques doivent être compétents et suffisamment abondants au moment où le virus est introduit, afin d'en assurer efficacement la diffusion ;

- la population humaine locale doit être hautement réceptive au virus introduit, c'est-à-dire immunologiquement vierge ou presque.

Ces différentes conditions ont été clairement remplies dans le sud de l'océan Indien, en 2005 [14,32] et dans le Nord-est de l'Italie, en 2007 [12,52], où $A$. albopictus s'est révélé un vecteur particulièrement efficace [55]. Par contre, elles n'étaient probablement pas entièrement remplies dans le midi de la France en 2010 [6,40], les mesures sanitaires prises sur place ayant pu aussi limiter les risques de diffusion locale.

\section{Essais de quantification de la virémie dans les infections inapparentes par DENV, CHIKV et ZIKV}

Au cours des épidémies, le nombre d'infections inapparentes est élevé, mais faute d'enquêtes adéquates, on ne connaît pas le niveau de la virémie qui les accompagne. On est donc contraint, pour estimer la virémie des formes inapparentes 
ou paucisymptomatiques, de faire appel aux données chiffrées concernant les infections apparentes.

Ainsi, pendant la grave épidémie de dengue qui a frappé la Polynésie française, en 1996 et 1997, les titres plasmatiques du DENV 2 étaient compris entre 1,7 et 5,6 $\log _{10} \mathrm{TCID}_{50} / \mathrm{ml}$, chez les enfants hospitalisés et la durée moyenne de la virémie était de 4,4 jours [44]. Une autre enquête réalisée chez 80 enfants thaillandais, au moyen d'une technique de RT-PCR capable de quantifier chacun des quatre sérotypes du DENV, a mis en évidence des taux plasmatiques d'ARN viral compris entre $5 \times 10^{3}$ et $10^{9}$ copies $/ \mathrm{ml}$ [62]. À Singapour, les autorités sanitaires ont estimé que les taux plasmatiques d'ARN viral des DENV se situaient entre $10^{5,5}$ et $10^{9,3}$ copies/ml [70]. Ces mêmes autorités sanitaires pensent « qu'il est probable, mais non prouvé, que les virémies sont plus faibles et de plus courte durée chez les sujets asymptomatiques que chez ceux qui présentent des symptômes »[70]. En l'absence de toute étude systématique publiée sur ce problème, cette opinion n'est qu'un postulat restant à démontrer.

Par contre, il paraît raisonnable d'avancer l'hypothèse qu'au moins certains cas d'infections inapparentes peuvent s'accompagner de virémies au moins aussi élevées que la moyenne des taux établis dans les infections apparentes. En effet, d'après les données de la littérature, les taux de virémie chez les patients souffrant de formes symptomatiques de dengue sont éminemment variables, d'une épidémie à une autre et d'une région géographique à une autre. Cette variabilité a de grandes chances d'exister aussi dans les formes inapparentes de la dengue, représentant un risque non négligeable d'introduction de cette virose dans une région non endémique.

Ce concept s'est d'ailleurs révélé correct dans les infections à CHIKV. Alors que des virémies aussi élevées que $3,3 \times 10^{9}$ copies/ml d'ARN viral ont été décelées chez des malades [47], plusieurs formes inapparentes ou cliniquement très légères ont été observées en Europe [12,47], en Australie [19] et en Amérique du Nord [36]. Ces individus rentraient de l'océan Indien ou d'Asie, où justement des épidémies de CHIKV étaient en cours.

On dispose de peu d'informations sur la virémie au cours des infections à ZIKV. Lors de l'épidémie de l'Île Yap, qui ressemblait à une épidémie de dengue, des titres d'ARN viral de $9 \times 10^{2}$ à $7,3 \times 10^{5}$ copies $/ \mathrm{ml}$ ont été observés. Chez un malade, ce titre était encore de $3,4 \times 10^{5}$, au $11^{\mathrm{e}}$ jour de la maladie [37].

\section{Essai d'appréciation du risque épidémiologique représenté par les formes inapparentes au cours des zoonoses arbovirales transmises par des moustiques}

En plus de l'Homme, les zoonoses arbovirales impliquent une grande variété d'hôtes vertébrés, sauvages ou domesti- ques, ainsi que de nombreux vecteurs. Par exemple, le cycle enzootique de l'encéphalite japonaise comprend des oiseaux aquatiques comme réservoirs, des porcs domestiques comme hôtes amplificateurs et une grande diversité de moustiques vecteurs [22]. L'Homme est un hôte accidentel pour le JEV et, comme chez lui la virémie est transitoire et de faible amplitude, il est incapable de transmettre le virus à d'autres humains ou à d'autres espèces animales [59]. Ainsi, à partir de 1995, le JEV a été introduit à plusieurs reprises en Australie, à partir de la Papouasie-Nouvelle-Guinée. On ne pense pas que ces introductions aient été réalisées par des malades virémiques, mais plus vraisemblablement par des moustiques infectés portés par les vents $[28,29,53,65,66]$.

Les infections à WNV sont également des zoonoses. Le WNV est capable d'infecter une grande variété d'oiseaux aquatiques et terrestres, des chevaux, des carnivores, des petits rongeurs, des alligators, ainsi qu'un très grand nombre de moustiques et de tiques [15]. Les infections humaines sont accidentelles et surviennent lorsqu'un moustique ornithophile infecté se gorge sur un Homme plutôt que sur un animal. Le WNV a été introduit aux États-Unis, probablement au début de 1999 et depuis le Moyen-Orient, mais les modalités exactes de son introduction ne sont pas connues. Il pouvait s'agir d'un oiseau, d'un moustique ou d'un homme infecté, transporté par avion. Puisque les infections humaines à WNV sont considérées comme des impasses épidémiologiques, il est en fait peu probable que le responsable de cette introduction ait été un voyageur infecté [64]. En Amérique du Nord, on sait que de nombreuses personnes ont été infectées de façon inapparente $[30,42,67,71]$ et que des contaminations humaines ont été induites par la transfusion sanguine, la transplantation d'organes, l'hémodialyse et, peut-être l'allaitement au sein [31]. Dans tous ces cas, un donneur asymptomatique a pu être à l'origine de la contamination. Enfin, l'infection humaine par le WNV peut être préclinique, n'attirant pas l'attention, tout en s'accompagnant d'une forte virémie [71].

En ce qui concerne l'émergence de l'USUV en Europe centrale, en 2001, ce sont probablement des oiseaux migrateurs qui sont à l'origine de son introduction. Il est maintenant bien implanté dans les faunes culicidiennes et aviaires autrichiennes [69].

Dans la fièvre de la Vallée du Rift, il n'y a pas de transmission interhumaine. Il est donc très peu probable que le RVFV ait été introduit en Péninsule arabique par un voyageur virémique. C'est plutôt par le bétail infecté ou des moustiques portés par le vent que cette région du monde a pu être contaminée [38].

\section{Conclusion}

Qu'il s'agisse d'infections apparentes ou inapparentes, il existe plusieurs mécanismes efficaces permettant à des 
arbovirus transmis par des moustiques de s'introduire puis de diffuser dans des régions géographiques jusque-là restées indemnes. Mais, le risque représenté, en propre, par les infections inapparentes ne peut plus rester ignoré : elles sont les plus nombreuses et nous n'avons aucun moyen de les détecter.

Remerciements : Ma gratitude va au Pr Ernest A. Gould, d'Oxford, Royaume-Uni, pour ses précieux conseils.

Conflit d'intérêt : l'auteur déclare ne pas avoir de conflit d'intérêt.

\section{Références}

1. Abu-Elyazeed R, el-Sharkawi S, Olson J, et al (1996) Prevalence of anti-Rift-Valley fever IgM antibody in abattoir workers in the Nile delta during the 1993 outbreak in Egypt. Bull World Health Organ 74(2): 155-8

2. Anderson SG, Donnelley M, Stevenson WJ, et al (1952) Murray Valley encephalitis: surveys of human and animal sera. Med J Aust 1(4):110-4

3. Arthur RR, el-Sharkawi S, Cope SE, et al (1993) Recurrence of Rift Valley fever in Egypt. Lancet 342(8880):1149-50

4. Balmaseda A, Hammond SN, Tellez Y, et al (2006) High seroprevalence of antibodies against dengue virus in a prospective study of schoolchildren in Managua, Nicaragua. Trop Med Int Health 11(6):935-42

5. Bessaud M, Peyrefitte CN, Pastorino BAM, et al (2006) Chikungunya strains, Reunion Island outbreak. Emerg Infect Dis 12(10): 1604-6

6. Bracq $J$ (27 septembre 2010) Un deuxième cas de chikungunya sur la Côte d'Azur. Le Figaro : 13

7. Brody JA, Burns KF, Browning G, Schattner JD (1959) Apparent and inapparent attack rates for St Louis encephalitis in selected population. New Engl J Med 261:644-6

8. Burke DS, Nisalak A, Johnson DE, Scott RM (1988) A prospective study of dengue infection in Bangkok. Am J Trop Med Hyg 38(1):172-80

9. Calzolari M, Bonilauri P, Bellini R, et al (2010) Evidence of simultaneous circulation of West Nile and Usutu viruses in mosquitoes sampled in Emilia-Romagna region (Italy) in 2009. PLoS One 5(12):e14324

10. CDC (2009) Japanese encephalitis among three US travelers returning from Asia, 2003-2008. MMWR Morb Mortal Wkly Rep 58(27):737-40

11. Charrel RN, de Lamballerie X, Raoult D (2007) Chikungunya outbreaks-The globalization of vectorborne diseases. N Engl J Med 356(8):769-71

12. Charrel RN, de Lamballerie X, Raoult D (2008) Seasonality of mosquitoes and chikungunya in Italy. Lancet Infect Dis 8(1):5-6

13. Chastel C (2002) Incidence des changements climatiques planétaires sur les arboviroses transmises à l'Homme par des moustiques et des tiques. Bull Acad Natl Med 186:89-101

14. Chastel C (2005) Le virus chikungunya : son extension récente à l'Île de la Réunion. Bull Acad Natl Med 189:1827-35

15. Chastel C (2007) Les virus bougent : périls planétaires. Bull Acad Natl Med 191:1563-77

16. Chastel C (2007) Virus et émergences virales dans un contexte de mondialisation : le grand défi. Med Trop 67(3):213-4
17. Chastel C (2008) Yellow fever, Historical. In: K Heggenhongen, $\mathrm{S}$ Quah (eds) International Encyclopedia of Public Health, Academic Press, San Diego 6:665-75

18. Downs WG, Shope RE, Kerr JA (1981) Yellow fever. In: Spittel JA, Clinical Medicine, Harper \& Row (eds) 1-27

19. Druce JD, Johnson DF, Tran T, et al (2007) Chikungunya virus infection in traveller to Australia. Emerg Infect Dis 13(3):509-10

20. Duffy MR, Chen TH, Hancock WT, et al (2009) Zika virus outbreak on Yap Island, Federated States of Micronesia. New Engl J Med 360(24):2536-43

21. Endy TP, Chunsuttiwat S, Nisalak A, et al (2002) Epidemiology of inapparent and symptomatic acute dengue virus infection: a prospective study of primary school children in Kamphaeng Phet, Thailand. Am J Epidemiol 156(1):40-51

22. Erlanger TE, Weiss S, Keiser J, et al (2009) Past, present and future of Japanese encephalitis. Emerg Infect Dis 15(1):1-7

23. Fritel X, Rollot O, Gérardin P, et al (2010) Chikungunya virus infection during pregnancy, Réunion, France, 2006. Emerg Infect Dis 16(3):418-25

24. Gould EA, Higgs S (2009) Impact of climate change and other factors on emerging arbovirus diseases. Trans R S Trop Med Hyg 103(2):109-21. Epub 2008

25. Halstead SB, Grosz CR (1962) Subclinical Japanese encephalitis. I. Infections of Americans with limited residence in Korea. Am J Hyg 75:190-201

26. Halstead SB (2006) Dengue in the Americas and Southeast Asia: do they differ? Rev Panam Salud Publica 20(6):407-15

27. Halstead SB (2007) Dengue. Lancet 370(9599):1644-52

28. Hanna JN, Ritchie SA, Phillips DA, et al (1996). An outbreak of Japanese encephalitis in Torres Strait, Australia, 1995. Med J Austr 165(5):256-60

29. Hanna JN, Ritchie SA, Phillips DA, et al (1999) Japanese encephalitis in North Queensland, Australia, 1998. Med J Austr 170 (11):533-6

30. Hayes EB, Sejvar JJ, Zaki SR, et al (2005) Virology, pathology, and clinical manifestations of West Nile virus disease. Emerg Infect Dis 11(8):1174-9

31. Hayes EB, Komar N, Nasci RS, et al (2005) Epidemiology and transmission dynamics of West Nile virus disease. Emerg Infect Dis 11(8):1167-73

32. Higgs S (2006) The 2005-2006 Chikungunya epidemic in the Indian Ocean. Vector Borne Zoonotic Dis 6(2):115-6

33. Jouan A, Adam F, Riou O, et al (1990) Évaluation des indicateurs de santé dans la région du Trarzo lors de l'épidémie de fièvre de la Vallée du Rift en 1987. Bull Soc Pathol Exot 83(5):621-7

34. Kaur P, Ponniah M, Murhekar MV, et al (2008) Chikungunya outbreak, South India, 2006. Emerg Infect Dis 14(10):1623-5

35. Lanciotti RS, Roehrig JT, Deubel V, et al (1999) Origin of the West Nile virus responsible for an outbreak of encephalitis in the northeastern United States. Science 286(5448):2333-7

36. Lanciotti RS, Kosoy OL, Laven JJ, et al (2007) Chikungunya virus in US travelers returning from India, 2006. Emerg Infect Dis 13(5):764-7

37. Lanciotti RS, Kosoy OL, Laven JJ, et al (2008) Genetic and serologic properties of Zika virus associated with an epidemic, Yap State, Micronesia, 2007. Emerg Infect Dis 14(8):1232-9

38. Madani TA, Al-Mazrou YY, Al-Jeffri MH, et al (2003) Rift Valley fever epidemic in Saudi Arabia: epidemiological, clinical, and laboratory characteristics. Clin Infect Dis 37(8):1084-92. Epub 2003

39. Meegan JM, Hoogstraal H, Moussa MI (1979) An epizootic of Rift Valley fever in Egypt in 1977. Vet Rec 105(6):124-5

40. Ministère de la Santé et des Sports (2010) Premier cas de dengue autochtone en métropole. http:// www.destinationsante.com/

41. Morvan J, Saluzzo JF, Fontenille D, et al (1991) Rift Valley fever on the east coast of Madagascar. Res Virol 142(6):475-82 
42. Mostashari F, Bunning ML, Kitsutani PT, et al (2001) Epidemic West Nile encephalitis, New York, 1999: results of a householdbased seroepidemiological survey. Lancet 358(9278):261-4

43. Mudur G (2006) Failure to control mosquitoes has led to two fever epidemics in India. BMJ 333(7572):773

44. Murgue B, Roche C, Chungue E, Deparis X (2000) Prospective study of the duration and magnitude of viraemia in children hospitalised during the 1996-1997 dengue-2 outbreak in French Polynesia. J Med Virol 60(4):432-8

45. Nemes Z, Kiss G, Madarassi EP, et al (2004) Nosocomial transmission of dengue. Emerg Infect Dis 10(10):1880-1

46. Ostlund MR, Kan B, Karlsson M, Vene S (2004) Japanese encephalitis in a Swedish tourist after travelling to Java and Bali. Scand J Infect Dis 36(6-7):512-3

47. Parola P, de Lamballerie X, Jourdan J, et al (2006) Novel chikungunya virus variant in travelers returning from Indian Ocean islands. Emerg Infect Dis 12(10):1493-8

48. Pialoux G, Gaüzère BA, Jauréguiberry S, Strobel M (2007) Chikungunya, an epidemic arbovirosis. Lancet Infect Dis 7(5):319-27

49. Queyriaux B, Simon F, Oliver M, Boutin JP (2008) Chikungunya et autres arboviroses en milieu tropical. Med Trop 68(5):478-81

50. Ravi V (2006) Re-emergence of chikungunya virus in India. Indian J Med Microbiol 24(2):83-4

51. Reiter P (2001) Climate change and mosquito-borne disease. Environ Health Perspect 109(Suppl 1):141-61

52. Rezza G, Nicoletti L, Angelini R, et al (2007) Infection with chikungunya virus in Italy: an outbreak in a temperate region. Lancet 370(9602):1840-6

53. Ritchie SA, Phillips D, Broom A, et al (1997) Isolation of Japanese encephalitis virus from Culex annulirostris in Australia. Am J Trop Med Hyg 56(1):80-4

54. Saluzzo JF, Digoutte JP, Chartier C, et al (1987) Focus of Rift Valley fever virus transmission in southern Mauritania. Lancet 1 (8531):504

55. Schuffenecker I, Iteman I, Michault A, et al (2006) Genome microevolution of chikungunya viruses causing the Indian Ocean outbreak. PloS Med 3(7):e263. Epub 2006

56. Shu PY, Chien LJ, Chang SF, et al (2005) Fever screening at airports and imported dengue. Emerg Infect Dis 11(3):460-2

57. Sissoko D, Renault P, Pierre V (2008) Chikungunya à Mayotte : caractéristiques cliniques et implications pour la surveillance épidémiologique. Bull Soc Pathol Exot 101:372-5 [http://www. pathexo.fr/documents/articles-bull/T101-4-3243-4p.pdf]
58. Sissoko D, Giry C, Gabrie P, et al (2009) Rift Valley fever, Mayotte, 2007-2008. Emerg Infect Dis 15(4):568-70

59. Solomon T (2006) Control of Japanese encephalitis-within our grasp? N Engl J Med 355(9):865-71

60. Southam CM (1956) Serological studies of encephalitis in Japan. II. Inapparent infections by Japanese B encephalitis virus. J Infect Dis $99(2): 163-9$

61. Southam CM (1956) Serological studies of encephalitis in Japan. III. Epidemic encephalitis other than Japanese B encephalitis in Tokyo. J Infect Dis 99(2):170-3

62. Sudiro TM, Zivny J, Ishiko H, et al (2001) Analysis of plasma viral RNA levels during acute dengue virus infection using quantitative competitor reverse transcription-polymerase chain reaction. J Med Virol 63(1):29-34

63. Tran A, Chastel C (2008) Grossesse et arbovirus transmis par des moustiques : conséquences pathologiques pour la mère et l'enfant. Une revue générale. Bull Soc Pathol Exot 101(5):418-23 [http://www.pathexo.fr/documents/articles-bull/T101-5-3246.pdf]

64. Tyler KL (2001) West Nile virus encephalitis in America. N Engl J Med 344(24):1858-9

65. Van Den Hurk AF, Montgomery BL, Northill JA, et al (2006) Short report: the first isolation of Japanese encephalitis virus from mosquitoes collected from mainland Australia. Am J Trop Med Hyg 75(1):21-5

66. Van den Hurk AF, Ritchie SA, Johansen CA, et al (2008) Domestic pigs and Japanese encephalitis virus infection, Australia. Emerg Infect Dis 14(11):1736-8

67. Weiss D, Carr D, Kellachan J, et al (2001) Clinical findings of West Nile virus infection in hospitalized patients, New York and New Jersey, 2000. Emerg Infect Dis 7(4):654-8

68. Weissenböck H, Kolodziejek J, Url A, et al (2002) Emergence of Usutu virus, an African mosquito-borne Flavivirus of the Japanese encephalitis virus group, central Europe. Emerg Infect Dis $8(7): 652-6$

69. Weissenböck H, Kolodziejek J, Url A, et al (2003) Usutu virus activity in Austria, 2001-2002. Microbes Infect 5(12):1132-6

70. Wilder-Smith A, Chen LH, Massad E, Wilson ME (2009) Threat of dengue to blood safety in dengue-endemic countries. Emerg Infect Dis 15(1):8-11

71. Zou S, Foster GA, Dodd RY, et al (2010) West Nile fever characteristics among viremic persons identified through blood donor screening. J Infect Dis 202(9):1354-61 